Orthopäde 2011 · 40:963-963

DOI 10.1007/s00132-011-1823-5

Online publiziert: 21. Oktober 2011

(c) Springer-Verlag 2011

\author{
B. Hintermann \\ Klinik für Orthopädie und Traumatologie des Bewegungsapparates, Kantonsspital Liestal
}

\title{
Sprunggelenkprothetik in der Schweiz
}

sprechende Ansätze für nachhaltige Korrekturen entwickelt. Die beiden Beiträge von Markus Knupp (Varusarthrose) und Arno Frigg (Valgusarthrose) zeigen in exzellenter Weise die komplexen Lösungsansätze für eine erfolgreiche Prothesenversorgung des deformierten und instabilen Sprunggelenks auf. Größere Knochendefekte werden ebenfalls gemeinhin als Kontraindikation für eine Endoprothese gesehen. Wie in berechtigten Einzelfällen eine Lösung gefunden werden kann, zeigt der Beitrag von Christoph Lampert sehr anschaulich auf. Ein besonderes Problem ist schließlich die schlechte Knochenqualität. So überrascht nicht, dass in den Anfängen die Ergebnisse der Endoprothetik beim Patienten mit chronischer Polyarthritis katastrophal waren und dementsprechend allgemein davon abgeraten wurde. Die ermutigenden Ergebnisse von Pascal Rippstein decken sich mit den eigenen Erfahrungen. Dieser ausgezeichnete Beitrag verdeutlicht sehr, dass die heutigen Prothesendesigns und operativen Techniken sich gerade bei diesen „Low-demanding"-Patienten hervorragend als bewegliche Lösung anbieten.

Ein nicht gelöstes Problem schien lange das mediale Schmerzsyndrom nach einer Sprunggelenkprothesenimplntation. Manche Prothese wurde mit der Diagnose „mediales Impingement“ erfolglos débridiert oder gar ausgebaut. Minutiöse Langzeitbeobachtungen und biomechanische Analysen, aber auch neuere Erkenntnisse im Balancieren des Rückfußes ermöglichten in den letzten Jahren, den medialen Schmerz in diesem Patientenkollektiv besser zu verstehen. Der profunde Beitrag von Alexej Barg gibt einen Einblick in den heutigen Wissenstand.

Eine gescheiterte Prothesenimplantation kann letztlich immer in eine Arthrodese münden. Häufig handelt es sich um Patienten jüngeren Alters. Manchmal sind die Nachbargelenke von arthrotischen Veränderungen betroffen oder sogar bereits arthrodesiert. Der eigene Beitrag fasst unsere Erfahrung mit der Revisionsarthroplastik des oberen Sprunggelenks zusammen. Die ermutigenden Ergebnisse lassen eine ähnliche Entwicklung wie für das Knie- und Hüftgelenk erhoffen, wo die Revisionsarthroplastik heute einen festen Stellenwert hat. Die Arthrodese nach gescheiterter Prothesenimplantation selbst ist nicht immer einfach, insbesondere wenn der Knochenverlust erheblich ist. Der Beitrag von Norman Espinosa fasst die heutigen Möglichkeiten in ausgezeichneter Weise zusammen.

Ich möchte den Autoren für Ihr riesiges Engagement, welches sie in die zeitlich sehr beschränkte Vorbereitung der Manuskripte steckten, herzlich danken. Des Weiteren möchte ich Dieter Kohn für das Vertrauen danken, als Gästeherausgeber für dieses Heft des Orthopäden dienen zu dürfen. Ich hoffe, dass unsere Anstrengungen letztlich unseren Patienten zugute kommen werden, indem sie über ein fundiertes Verständnis zu besseren Ergebnissen sowie zu einer höheren Lebensqualität beitragen.

Ihr

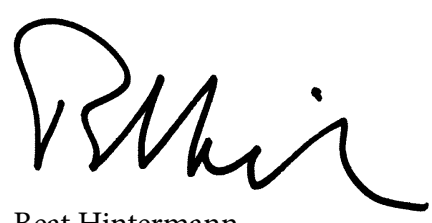

Beat Hintermann

\section{Korrespondenzadresse \\ Prof. Dr. B. Hintermann}

Klinik für Orthopädie und Traumatologie des Bewegungsapparates, Kantonsspital Liestal Rheinstraße 26, CH-4410 Liestal, Schweiz beat.hintermann@ksli.ch
Deformitäten und Instabilitäten des Rückfußes erkannt und es haben sich viel ver- 